\title{
Implementation of the 2018 American Society of Clinical Oncology/ College of American Pathologists Guidelines on HER2/neu Assessment by FISH in breast cancers: predicted impact in a single institutional cohort
}

\author{
Somaye Zare ${ }^{1} \cdot$ Juan Rong ${ }^{1} \cdot$ Svenja Daehne ${ }^{1}$ Andres Roma ${ }^{1} \cdot$ Farnaz Hasteh $^{1} \cdot$ Marie Dell'Aquila $^{1}$. \\ Oluwole Fadare ${ }^{1}$
}

Received: 26 February 2019 / Revised: 21 April 2019 / Accepted: 1 May 2019 / Published online: 12 June 2019

(c) United States \& Canadian Academy of Pathology 2019

\begin{abstract}
The 2018 American Society of Clinical Oncology (ASCO) and College of American Pathologists (CAP) update modified the interpretation guidelines for human epidermal growth factor receptor 2 (HER2) testing by incorporating immunohistochemistry (IHC) and fluorescence in situ hybridization (FISH) results in a subset of cases. Importantly, the new guidelines eliminate "equivocal" results, as well as the use of alternative chromosome 17 probes as the primary strategy for resolving the indeterminate FISH results. Herein, we investigate the predicted impact of implementing the 2018 ASCO/CAP guidelines on HER2 assessment by FISH in breast cancers, using data from a single institution. We compared the HER2 status of 1542 consecutive cases of breast carcinoma, interpreted by 2013 and 2018 ASCO/CAP guidelines. In total, $10.7 \%$ (165/1542) of the cases had a different final interpretation by 2018 guidelines compared with 2013 guidelines, including 70 previously HER2-positive cases reclassified as negative, four previously negative cases reclassified as positive, and 91 previously equivocal cases reclassified as negative. Overall, the number of HER2-positive cancers was reduced by 66 cases (4.3\% reduction in the HER2 positivity rate). The newly HER2-negative cases were mostly estrogen receptor positive $(90 \%)$, progesterone receptor positive $(80 \%)$, stage $1(60.9 \%)$, and grade $1-2(59.4 \%)$ cancers; $70 \%$ of them had been designated as HER2 positive only after the use of an alternative chromosome 17 FISH probe after an intially equivocal result from the standard CEP17 probe. Overall, implementing the revised 2018 HER2 guidelines is predicted to change the HER2 results of $10.7 \%$ of breast cancers, mainly by reclassifying previously equivocal to negative results.
\end{abstract}

\section{Introduction}

Human epidermal growth factor receptor 2 protein (HER2/ neu or HER2), encoded by the ERBB2 gene located on the long arm of chromosome 17 , is a transmembrane tyrosine kinase protein $[1,2]$. HER2 amplification and/or

Supplementary information The online version of this article (https:// doi.org/10.1038/s41379-019-0295-8) contains supplementary material, which is available to authorized users.

$\triangle$ Oluwole Fadare

ofadare@ucsd.edu

1 Department of Pathology, Anatomic Pathology Division, University of California San Diego Health, La Jolla, CA 92037, USA overexpression occurs in up to $20 \%$ of invasive breast cancers and is linked with aggressive disease and poor prognosis [3, 4]. Anti-HER2 therapies result in improved clinical outcomes in the patients with HER2-amplified tumors $[5,6]$. Accurate testing of HER2 status is important for making clinical decisions regarding patients' eligibility for targeted therapies and for predicting response to treatment [7]. The most commonly used tests for assessing HER2 status are immunohistochemistry (IHC) assays for HER2 protein expression and fluorescence in situ hybridization (FISH) for HER2 gene amplification.

In an effort to standardize the performance of HER2 testing, The American Society of Clinical Oncology (ASCO) and College of American Pathologists (CAP) released detailed guidelines for conducting and interpreting HER2 testing in breast cancers in 2007 [8] and updated those recommendations in 2013 [9]. Since then, several 
studies have evaluated the practical implications of the 2013 guidelines and additional data have been generated regarding the less common HER2 testing patterns based on clinical trials and other data sets. In 2018, updated ASCO/ CAP guidelines and recommendations for HER2 testing in breast cancers were published [10].

The 2018 update changes the diagnostic categories for HER2 status in several important ways by incorporating IHC and FISH results. Although the recommendations for classical HER2- positive (group $1=$ HER2:CEP17 ratio $\geq 2.0$ and HER2 copy number $\geq 4.0$ ) and classical HER2-negative (group 5 = HER2:CEP17 ratio <2.0 and HER2 copy number $<4.0$ ) breast cancers are similar to 2013 guidelines, significant changes are made in the interpretation of HER2 results in cancers with less common HER2 FISH results (group $2=$ HER2:CEP17 ratio $\geq 2.0$ and HER2 copy number $<4.0$, group $3=$ HER2:CEP17 ratio $<2.0$ and HER2 copy number $\geq 6.0$, and group $4=\mathrm{HER} 2$ :CEP17 ratio $<2.0$ and HER2 copy number 24.0 ) [10]. Importantly, the new guidelines eliminate the designation of "equivocal" in the final interpretation of HER2 FISH, which was recognized as a category in the prior versions, and recommends determining the HER2 status as "positive" or "negative" by correlating with IHC results and re-evaluation of FISH counts in cases of equivocal IHC results. In addition, the most recent guidelines no longer recommend employing reflex FISH testing by an alternative chromosome 17 probe [10].

The aim of our study was to investigate the potential impact of implementing the revised 2018 ASCO/CAP recommendations on the determination of HER2 status in breast cancers. We retrospectively re-evaluated HER 2 status based on 2018 guidelines and compared the results with our original interpretations by the 2013 ASCO/CAP guidelines.

\section{Materials and methods}

\section{Study cohort}

After approval from the Institutional Review Board at the University of California San Diego, 1542 consecutive cases of primary and metastatic breast carcinoma that underwent HER2 testing at our institution between January 2014 and December 2017 were identified from an electronic database. In all cases, HER2 testing was performed by both IHC and dual-colored HER2/CEP17 FISH probes, as is our institutional protocol $[11,12]$. During this time period, all aspects of specimen handling, including fixation times, cold ischemic times, as well as the HER2 IHC and FISH result interpretations followed CAP or ASCO/CAP 2013 guidelines [9]. The patient demographic data, pathologic tumor characteristics, HER2 IHC results, and HER2 FISH results were extracted from the pathology reports.

\section{HER2 IHC testing}

For the study period, all IHC HER2 analysis tests were performed on 4- $\mu$-thick sections of formalin-fixed, paraffin-embedded tissue using Ventana automation, the Ultra View detection kit, and a rabbit monoclonal antibody to HER2 (clone 4B5, prediluted, Ventana Medical Systems, Tucson, AZ). The intensity and the proportion of tumor cells stained were assessed on cytoplasmic membrane staining. All HER2 IHC results were interpreted and reviewed by a rotating group of six staff pathologists, all with specific experience in breast pathology.

Invasive breast cancers with $3+$ uniform intense membrane staining in $>10 \%$ of tumor cells were interpreted as HER2 IHC positive. An equivocal result was rendered when more than $10 \%$ of tumor cells showed weak-tomoderate cytoplasmic membrane staining. The results that did not fulfill the above criteria were considered negative.

\section{HER2 FISH testing}

FISH was performed in formalin-fixed, paraffin-embedded target tissue. Hematoxylin and eosin (H\&E)-stained sections and HER2 IHC slides were evaluated by a pathologist to label the invasive carcinoma. FISH analyses were performed using dual-color HER2/CEP17 assay (PathVysion Probe Kit; Abbott Molecular, Abbott Park, IL). FISH signals were analyzed and captured using the CytoVision software (Leica Biosystems Richmond, Inc., Richmond, IL, USA). HER2 and CEP17 signals were manually counted by two technologists; each technologist scored at least 20 tumor cell nuclei independently and calculated HER2/ CEP17 ratios, blinded to the other technologist's results. In rare cases of discordant results, the third and fourth technologists reanalyzed the slides and calculated another independent HER2/CEP17 ratio (for detailed information and counts, see Supplementary Table S1). The final results were interpreted by a cytogeneticist (M.D.'A.), and were reviewed and finalized by a breast pathologist. The original interpretation and reports were based on 2013 ASCO/CAP criteria [9]. For the study period, repeat testing with alternative chromosome 17 probes (LIS1/RARA [Vysis MillerDieker Region/Isolated Lissencephaly Probe LSI LIS1/LSI RARA; Abbott Molecular, Abbott Park, IL]) was performed for stratification of equivocal results (group 4), according to the 2013 ASCO/CAP recommendations.

For the purpose of this study, FISH data, including the number of nuclei scored, HER2 and CEP17 signal counts, each cyrotechnologist's results, and HER2/CEP17 ratio were reviewed. HER2 status was reclassified using the 2018 ASCO/CAP guidelines by incorporating HER2 IHC and FISH data [10]. No renewed FISH count was performed in group 2-4 cases with equivocal IHC results, because all the 
FISH cases are routinely counted by at least two independent observers in our institution. The results of 2018 update guidelines were compared with the original HER2 status data based on 2013 ASCO/CAP criteria.

\section{Results}

During the study period, a total of 1542 breast carcinomas underwent dual-probe FISH analysis for HER2 gene status in our institution. The specimens were from 1391 women and 4 men with a mean age of 57.6 years (range 22-99 years). In the original designation by 2013 guidlines, there were 269 (17.4\%) positive, 1178 (76.4\%) negative, and 95 (6.2\%) equivocal HER2 FISH results. Whereas by using the 2018 scheme, when IHC results were incorporated in cases from less common groups (2-4), there were 1339 (86.8\%) negative cases, 203 (13.2\%) positive results, and no equivocal interpretations (Table 1).

For the purpose of this study, all the cases were assigned to different FISH groups [10,13] as follows: group 1: 189 $(12.26 \%)$, group 2: $23(1.5 \%)$, group 3: $8(0.5 \%)$, group 4 : $144(9.34 \%)$, and group 5: 1178 (76.4\%). The data for each category as well as changes that were observed by the new recommendations in each group are explained in detail in the following sections, and the findings are summarized in Table 2.

Table 1 Comparison of FISH results by 2013 and 2018 ASCO/CAP guidelines

\begin{tabular}{lccc}
\hline 2013 guidelines & \multicolumn{2}{l}{2018 guidelines, No. $(\%)$} & \multirow{2}{*}{ Total } \\
\cline { 2 - 3 } & Negative & Positive & \\
\hline Negative & $1178(76.4 \%)$ & 0 & $1178(76.4 \%)$ \\
Equivocal & $91(5.9 \%)$ & $4(0.2 \%)$ & $95(6.2 \%)$ \\
Positive & $70(4.5 \%)$ & $199(12.9 \%)$ & $269(17.4 \%)$ \\
Total & $1339(86.8 \%)$ & $203(13.2 \%)$ & $1542(100 \%)$ \\
\hline
\end{tabular}

\section{Group 1 (HER2:CEP17 ratio $\geq 2.0$ and HER2 copy number $\geq 4.0$ )}

In total, $189(12.26 \%)$ cases showed HER2:CEP17 ratio $\geq 2.0$ and HER 2 copy number $\geq 4.0$ and were interpreted as HER 2 positive by both 2013 and 2018 guidelines. The majority (173 of $189,91.5 \%$ ) of the cases in this group had $2+$ or $3+$ immunostaining results.

\section{Group 2 (HER2:CEP17 ratio $\geq 2.0$ and HER2 copy number <4.0)}

All $23(1.5 \%)$ cases in this group were interpreted as HER2 positive by 2013 guidelines. ASCO/CAP 2018 guidelines classify the group 2 cases with positive IHC $(3+)$ as HER2 positive and all the cases with negative IHC are considered HER2 negative. In cases with $2+$ IHC staining, recounting at least 20 cells FISH by an additional observer, blinded to previous results is recommended. If FISH recount shows similar results, HER2 is reported as negative with a comment discussing the limited evidence on the efficacy of HER2-targeted therapy in the cancers from this group in the absence of HER2 protein overexpression.

In our study, 14 of these 23 cases had negative IHC and nine cases showed $2+$ staining. None of the cases in this group had positive IHC results as reported by our previous study [14]; therefore, by 2018 recommendations, all 23 cases were interpreted as negative (Fig. 1a).

\section{Group 3 (HER2:CEP17 ratio $<2.0$ and HER2 copy number $\geq 6.0$ )}

Only eight $(0.5 \%)$ cases belonged to this group, which were interpreted as HER 2 amplified by the 2013 scheme. By 2018 criteria, group 3 cases with negative $(0,1+)$ IHC are designated as HER 2 negative and the cases with $3+$ are considered HER 2 positive. In cases with $2+$ IHC staining, FISH recount by an additional observer, blinded to previous results is recommended. If FISH recount shows similar results, HER2 is reported as positive.
Table 2 Classification of HER2 result groups by 2013 and 2018 guidelines

\begin{tabular}{|c|c|c|c|c|c|c|}
\hline \multirow[t]{2}{*}{ HER2 FISH groups } & \multirow[t]{2}{*}{ Group definitions } & \multicolumn{3}{|c|}{ HER2 by ASCO/CAP 2013} & \multicolumn{2}{|c|}{$\begin{array}{l}\text { HER } 2 \text { by ASCO/ } \\
\text { CAP } 2018\end{array}$} \\
\hline & & Positive & Negative & Equivocal & Positive & Negative \\
\hline Group $1(n=189)$ & Ratio $\geq 2.0$, HER $2 \geq 4.0$ & 189 & 0 & 0 & 189 & 0 \\
\hline Group $2(n=23)$ & Ratio $\geq 2.0$, HER $2<4.0$ & 23 & 0 & 0 & 0 & 23 \\
\hline Group $3(n=8)$ & Ratio $<2.0$, HER2 $\geq 6.0$ & 8 & 0 & 0 & 6 & 2 \\
\hline Group $4(n=144)$ & Ratio $<2.0$, HER2 $\geq 4.0$ & 49 & 0 & 95 & 8 & 136 \\
\hline Group $5(n=1178)$ & Ratio $<2.0$, HER $2<4.0$ & 0 & 1178 & 0 & 0 & 1178 \\
\hline Total $(n=1542)$ & & 269 & 1178 & 95 & 203 & 1339 \\
\hline
\end{tabular}



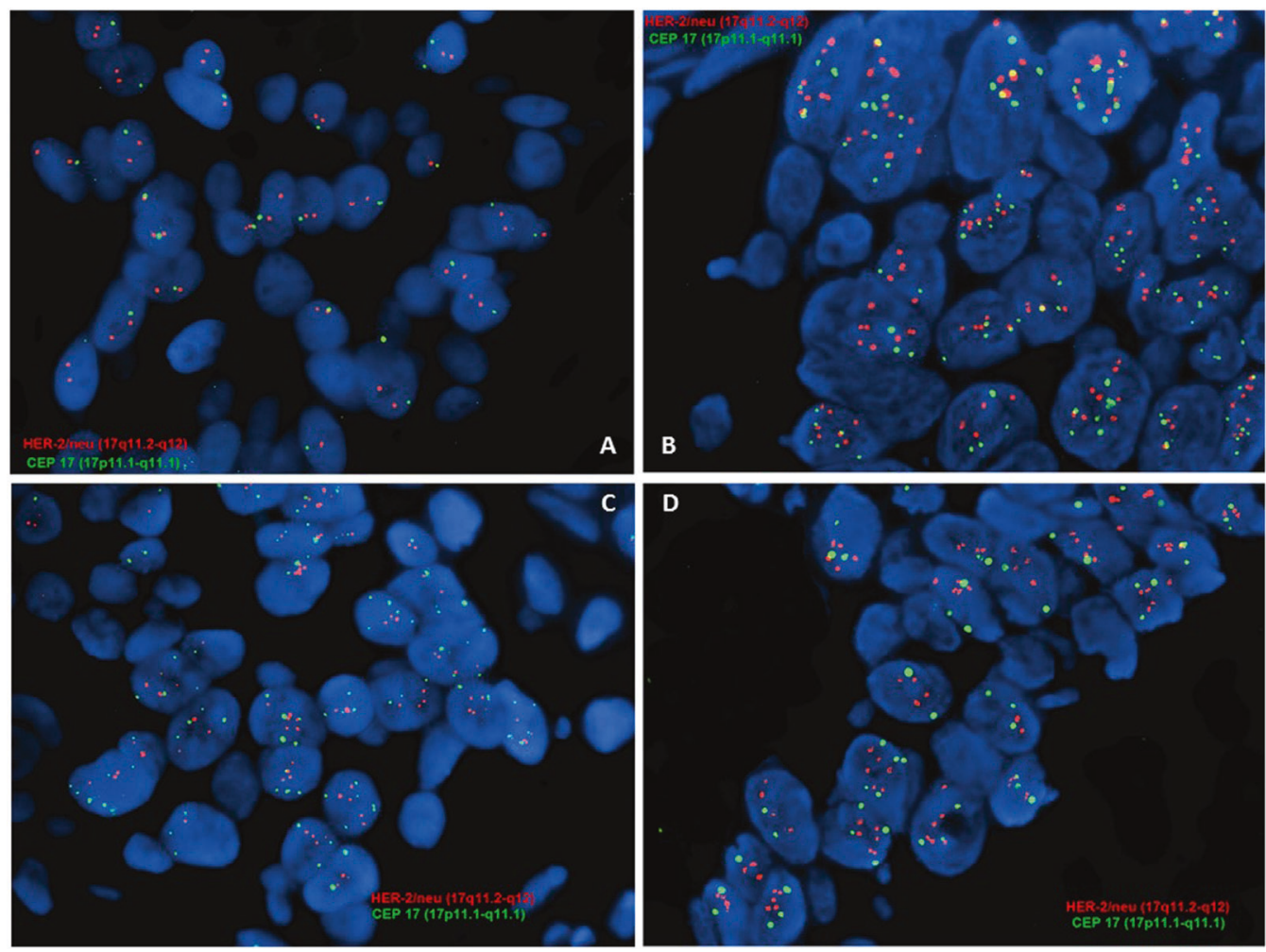

Fig. 1 Examples of HER2 fluorescence in situ hybridization (FISH) images corresponding to breast cancers, which were reclassified by 2018 ASCO/CAP guidelines. HER2, Spectrum Orange and CEP17, Spectrum Green. a A case from group 2: HER2/CEP17 ratio $\geq 2.0$; average HER2 copy number $<4.0$ with a negative HER2 immunohistochemical stain (IHC), classified as negative by 2018 guidelines. b A case from group 3: HER2/CEP17 ratio <2.0; average HER2 copy

Within these eight cases, two were IHC negative, three were IHC $2+$, and three showed positive IHC results. According to 2018 recommendations, six (75\%) cases were classified as positive and two (25\%) as negative (Fig. 1b).

\section{Group 4 (HER2:CEP17 ratio $<2.0$ and HER2 copy number $\geq 4.0$ )}

By 2013 criteria, this group of breast cancers was interpreted as equivocal and the guidelines allowed for the use of additional testing by an alternative chromosome 17 probe to stratify the results. Originally, among the $144(9.3 \%)$ breast cancers in this group, 49 (34\%) cases were classified as HER2 amplified upon testing with the alternative probe and $95(66 \%)$ remained equivocal.

The updated 2018 guidelines no longer recommend the use of reflex testing with alternative probes and eliminate the "equivocal" designation. The final determination of HER2 status in cases of this group is made in correlation with IHC results, with positive IHC as HER2 positive and negative IHC as HER2 negative. In cases with $2+$ IHC staining, recounting FISH by an additional observer,

number $\geq 6.0$ with negative IHC, classified as negative by 2018 guidlines. c A case from group 4: HER2/CEP17 ratio <2.0; average HER2 copy number $\geq 4.0$ and $<6.0$ with positive IHC, classified as positive by 2018 guidelines. d A case from group 4: HER2/CEP17 ratio <2.0; average HER2 copy number $\geq 4.0$ and $<6.0$ with equivocal IHC, classified as negative by 2018 guidelines

blinded to previous results is recommended. If FISH recount shows similar results, HER2 is reported as negative with a comment discussing the limited evidence on the efficacy of HER2-targeted therapy in the cancers from this group in the absence of HER2 protein overexpression.

Among 144 cases in this group, 8 (5.6\%) had positive IHC, $82(56.9 \%)$ had $2+$ staining, and $54(37.5 \%)$ were negative. Under the 2018 scheme, only eight (5.6\%) cases were classified as HER2 positive (Fig. 1c), four (50\%) of which were originally designated as HER 2 positive by using an alternative probe and the other four were interpreted as equivocal by reflex testing. In total, 136 cases (94.4\%) from this group were defined as negative by new guidelines (Fig. 1d), of which 91 were equivocal and 45 were positive (by an alternative chromosome 17 probe) by 2013 recommendations.

\section{Group 5 (HER2:CEP17 ratio <2.0 and HER2 copy number $<4.0$ )}

In total, $1178(76.4 \%)$ cases were interpreted as HER2 FISH negative by both 2013 and 2018 guidelines. The 


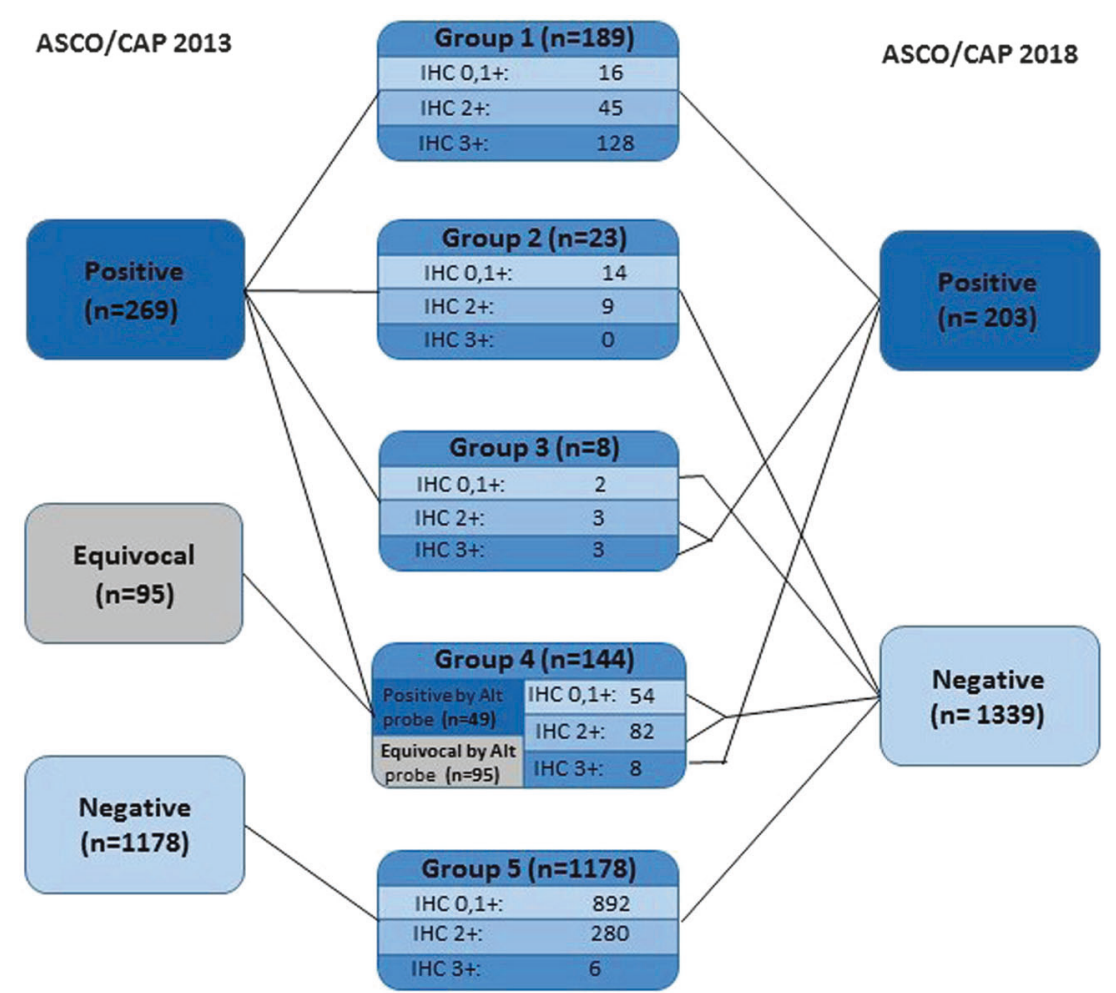

Fig. 2 HER2 fluorescence in situ hybridization (FISH) classification by 2013 and 2018 American Society of Clinical Oncology (ASCO)/ College of American Pathologists (CAP) guidelines. HER2 FISH

majority $(892,75.7 \%)$ of these cases had negative immunostaining results, 280 of them had IHC $2+$, and 6 cases were IHC $3+$.

\section{Comparison of HER2 status by 2018 vs. 2013 ASCO/ CAP guidelines}

In summary, of the 1542 breast cancers, 165 (10.7\%) cases had a different final interpretation by 2018 guidelines when compared with 2013 results. By the new guidelines, 70 HER2-positive cases were reclassified as negative, four equivocal cases became positive, and 87 equivocal cases became negative (Fig. 2). A total of 161 cancers were added to HER2-negative cases by the new guidelines (1339 vs. $1178,10.4 \%$ increase). Overall, the number of HER2positive cancers were reduced by 66 cases $(4.3 \%$ of all cases and $24.2 \%$ of HER2-positive cases).

\section{Cases with different HER2 interpretations by 2018 ASCO/CAP guidelines}

A review of the clinicopathologic characteristics of the 70 cases that were reclassified as HER 2 negative by the new guidelines showed that these cancers were mostly results as categorized by 2013 ASCO/CAP guidelines (left) and reclassified by 2018 ASCO/CAP guidelines (right)

estrogen receptor positive $(90 \%)$, progesterone receptor positive (80\%), stage $1(60.9 \%)$, and grade $1-2(59.4 \%)$ cancers (Table 3). The majority of the newly HER2/neunegative cases (45 cases, 64.3\%) had previously been classified as positive, due to the use of an alternative chromosome 17 probe on HER2-equivocal cases using 2013 ASCO/CAP criteria. Treatment and follow-up data were available for $62(88.6 \%)$ patients, $44(71 \%)$ of which had received HER2-targeted therapy (Supplementary Table 2). With a median follow-up of 36 months (range, 12-64 months) in the treated group, two patients experienced disease progression with metastatic disease and one of them was dead of disease. Similarly, in the untreated group, at a median of 36 months of follow-up (range, 6-63 months), one patient showed progression and was dead of disease on follow-up.

The new guidelines reclassified 87 previously equivocal cases as negative. Using 2013 guidelines, these group 4 cases had equivocal results by the HER2/CEP17 FISH probes and continued to have an equivocal result, even after the use of an alternative chromosome 17 probe. None of them received anti-HER2 therapy.

Four cases with previous equivocal HER2 FISH results were reclassified as HER2 positive by the new guidelines. 
Table 3 Clinicopathologic characteristics of breast cancers with HER2-positive results by 2013 guidelines, but reclassified as HER2 negative by 2018 ASCO/CAP guidelines

\begin{tabular}{lc}
\hline Tumor characteristics & Value \\
\hline Number of patients & 70 \\
Age (range [mean]) & $25-95(57.7)$ \\
Tumors types $(n[\%])$ & \\
Invasive ductal carcinoma & 52 \\
Invasive lobular carcinoma & 2 \\
Mixed ductal and lobular & 6 \\
Special types & 2 \\
NA & 8 \\
Histologic grade $(n[\%])$ & \\
G1 & $8(12.5)$ \\
G2 & $30(46.9)$ \\
G3 & $26(40.6)$ \\
NA & 6 \\
Pathologic stage $(n[\%])$ & \\
T1 & $39(60.9)$ \\
T2 & $19(29.7)$ \\
T3 & $6(9.4)$ \\
NA & 6 \\
Lymph node status $(n[\%])$ & $26(37.1)$ \\
Positive lymph nodes & $44(62.9)$ \\
Negative lymph nodes & 0 \\
NA & \\
Hormone status $(n[\%])$ & $28(45.2)$ \\
ER+ & $34(54.8)$ \\
ER- & 8 \\
PR+ & \\
PR- & $14(20)$ \\
HER2 IHC $(n[\%])$ & \\
Negative $(0,1+)$ & \\
Equivocal $(2+)$ & \\
Positive $(3+)$ & \\
\hline$G$ gras & \\
\hline &
\end{tabular}

$G$ grade, $N A$ not available, $T$ tumor stage

These four cases had been treated as a HER2-positive breast cancer and had received anti-HER2 therapy, based on positive IHC results.

\section{Discussion}

Several studies reported an increase in the number of equivocal cases following implementation of the $2013 \mathrm{ASCO} /$ CAP recommendations [15-17]. In 2018, ASCO/CAP updated their recommendations for HER2 testing, based on several cohort studies that reported their experiences with the 2013 guidelines. The revised guidelines include significant changes in HER2 testing interpretation for less common
HER2 FISH testing patterns, since more information had become available from the aforementioned studies regarding the frequency and prognostic characteristics of these groups. The new guidelines eliminate "equivocal" results, as well as the use of alternative chromosome 17 probes as the primary strategy for resolving these cases, given the limited evidence on the validity of this approach.

Our retrospective single institutional study demonstrated that although the status of $\sim 90 \%$ of our cases (groups 1 and 5) was not affected by implementing the 2018 ASCO/CAP guidelines, the new changes resulted in a significant reduction in HER2-positive interpretation compared with the 2013 scheme (203 vs. 269; 4.3\% of all cases). These results also highlight that most of the previously positive cases from the less common groups did not show HER2 protein overexpression by IHC.

In addition, the new guidelines eliminate the "equivocal" category and recommend against reflex testing, using an alternative chromosome 17 probe for initially equivocal cases. This single recommendation arguably had the most profound effect on our study results. Our data show that the majority (95.8\%) of 2013 FISH-equivocal cases were reclassified as negative, when the IHC results were incorporated into a final determination. Overall, the 2018 guidelines decrease the challenges in management of these patients by allowing for a definitive designation of HER2 status.

Among the newly HER2-negative cancers in this study, $32.8 \%(n=23)$ belong to group 2 . According to Press et al., group 2 patients showed no benefit from trastuzumab [18]. Only $2.9 \%(n=2)$ were from group 3 , which encompasses a heterogeneous group of breast cancers with limited and conflicting data $[13,18,19]$. The majority of the newly HER2-negative cases were from group 4, and had previously been classified as positive, due to the use of an alternative chromosome 17 probe to resolve initially equivocal results obtained from using the standard CEP17 centromeric probe. Many studies have reported their experience with this approach $[12,20,21]$ and in a recent study, Press et al. reported that the indiscriminate use of alternative controls may lead to false-positive interpretations of HER2 status, resulting from unrecognized heterozygous deletions in these alternative control genomic sites and incorrect HER2 ratio determinations [22]. In the new updates, the panel strongly recommended against this strategy as a routine practice.

In this study, most of the 70 newly HER2-negative breast cancers were hormone receptor positive, stage 1, and lowgrade cancers. The above findings indicate that these tumors show biological characteristics that are more similar to the profile that is expected for HER2-negative cancers, and seem to bolster the guidelines that they should be classified as HER2 negative. 
Our findings are by and large concordant with two recent studies, examining the impact of the new 2018 criteria. Liu et al., in a study from a Chinese institute, reported changes in classifications of HER2 status in $8.2 \%$ of the patients with breast cancer. These were mostly comprising the cases that were equivocal by 2013 criteria and became negative under the new updates. Only $0.4 \%$ of the cases were positive by previous guidelines and became negative by the 2018 version; however, they did not use an alternative chromosome 17 probe and all of their group 4 cases were equivocal by 2013 guidelines [23]. In another study on 331 breast cancers with equivocal IHC results, the new guidelines increased the rate of negative HER2 FISH results [24]. Murray et al. reported their experience with 2007, 2013, and 2018 versions of ASCO/CAP guidelines in a cohort of breast cancers, with equivocal HER2 results by IHC from Ireland. They suggested that the 2018 update heralds a potential change in therapeutic options for a significant number of patients, with $2.9 \%$ of FISH-positive tumors, according to 2007 and 2013 guidelines now categorized as HER2 negative [25].

Our single institutional retrospective study demonstrated that by implementation of 2018 ASCO/CAP guidelines, a significant number of patients with breast cancer $(10.7 \%$ in our study) would be classified differently compared with the original designations that were made using the 2013 iteration of those guidelines, potentially leading to different managements and outcomes. Additional studies with clinical outcome data are needed to assess the consequences of these changes in this subset of patients.

Funding This study was presented in part at the Annual Meeting of the United States and Canadian Academy of Pathology, National Harbor, Maryland, March 2019.

\section{Compliance with ethical standards}

Conflict of interest The authors declare that they have no conflict of interest.

Publisher's note: Springer Nature remains neutral with regard to jurisdictional claims in published maps and institutional affiliations.

\section{References}

1. Yarden Y. Biology of HER 2 and its importance in breast cancer. Oncology. 2001;61(Suppl. 2):1-13.

2. Yarden Y, Sliwkowski MX. Untangling the ErbB signalling network. Nat Rev Mol Cell Biol. 2001;2:127-37.

3. Owens MA, Horten BC, Da Silva MM. HER2 amplification ratios by fluorescence in situ hybridization and correlation with immunohistochemistry in a cohort of 6556 breast cancer tissues. Clin Breast Cancer. 2004;5:63-9.

4. Press MF, Bernstein L, Thomas PA, Meisner LF, Zhou JY, Ma Y, et al. HER-2/neu gene amplification characterized by fluorescence in situ hybridization: poor prognosis in node-negative breast carcinomas. J Clin Oncol 1997;15:2894-904.
5. Cobleigh MA, Vogel CL, Tripathy D, Robert NJ, Scholl S, Fehrenbacher L, et al. Multinational study of the efficacy and safety of humanized anti-HER2 monoclonal antibody in women who have HER2-overexpressing metastatic breast cancer that has progressed after chemotherapy for metastatic disease. J Clin Oncol. 2018;17:2639-48.

6. Vogel CL, Cobleigh MA, Tripathy D, Gutheil JC, Harris LN, Fehrenbacher L, et al. Efficacy and safety of Trastuzumab as a single agent. J Clin Oncol. 2002;20:719-26.

7. Jeffery S. Ross. The HER-2 / neu Gene and protein in breast cancer 2003: biomarker and target of therapy. Oncologist. 2003;8:307-25.

8. Wolff AC, Hammond ME, Schwartz JN, Hagerty KL, Allred DC, Cote RJ, et al. American Society of Clinical Oncology/College of American Pathologists guideline recommendations for human epidermal growth factor receptor 2 testing in breast cancer. J Clin Oncol. 2007;131:18-43.

9. Wolff AC, Hammond ME, Hicks DG, Dowsett M, McShane $\mathrm{LM}$, Allison $\mathrm{KH}$, et al. Recommendations for human epidermal growth factor receptor 2 testing in breast cancer: American Society of Clinical Oncology/College of American Pathologists clinical practice guideline update. J Clin Oncol. 2013; 31:3997-4013.

10. Wolff AC, Hammond MEH, Allison KH, Harvey BE, Mangu PB, Bartlett JMS, et al. Human epidermal growth factor receptor 2 testing in breast cancer: American society of clinical oncology/ college of American pathologists clinical practice guideline focused update. J Clin Oncol. 2018;36:2105-22.

11. Solomon JP, Dell'Aquila M, Fadare O, Hasteh F. Her2/neu status determination in breast cancer: a single institutional experience using a dual-testing approach with immunohistochemistry and fluorescence in situ hybridization. Am J Clin Pathol. 2017;147:432-7.

12. Zare S, Lin L, Alghamdi AG, Daehne S, Roma AA, Hasteh F, et al. Comparative pathologic analysis of breast cancers classified as HER2/NEU - amplified by fish using a standard HER2/cep17 dual probe and an alternative chromosome 17 control probe. Am J Surg Pathol. 2018;42:1208-15.

13. Press MF, Villalobos I, Santiago A, Guzman R, Cervantes M, Gasparyan A, et al. Assessing the new American Society of Clinical Oncology/College of American Pathologists guidelines for HER2 testing by fluorescence in situ hybridization: experience of an academic consultation practice. Arch Pathol Lab Med. 2016;140:1250-8.

14. Zare SY, Lin L, Alghamdi AG, Daehne S, Roma AA, Hasteh F, et al. Breast cancers with a HER2/CEP17 ratio of 2.0 or greater and an average HER 2 copy number of less than 4.0 per cell: frequency, immunohistochemical correlation, and clinicopathological features. Hum Pathol. 2019;83:7-13.

15. Lim TH, Lim AST, Thike AA, Tien SL, Tan PH. Implications of the Updated 2013 American Society of Clinical Oncology/College of American Pathologists Guideline Recommendations on human epidermal growth factor receptor 2 gene testing using immunohistochemistry and fluorescence in situ hybridization for breast cancer. Arch Pathol Lab Med. 2016;140:140-7.

16. Singh K, Tantravahi U, Lomme MM, Pasquariello T, Steinhoff M, Sung CJ. Updated 2013 College of American Pathologists/ American Society of Clinical Oncology (CAP/ASCO) guideline recommendations for human epidermal growth factor receptor 2 (HER2) fluorescent in situ hybridization (FISH) testing increase HER2 positive and HER2 e. Breast Cancer Res Treat. 2016; 157:405-11.

17. Long TH, Lawce H, Durum C, Moore SR, Olson SB, Gatter K, et al. The new equivocal: changes to HER2 FISH results when applying the 2013 ASCO/CAP guidelines. Am J Clin Pathol. 2015;144:253-62. 
18. Press MF, Sauter G, Buyse M, Fourmanoir H, Quinaux E, TsaoWei DD, et al. HER2 gene amplification testing by fluorescent in situ hybridization (FISH): comparison of the ASCO-College of American Pathologists guidelines with FISH scores used for enrollment in Breast Cancer International Research Group clinical trials. J Clin Oncol. 2016;34:3518-28.

19. Ballard M, Jalikis F, Krings G, Schmidt RA, Chen YY, Rendi $\mathrm{MH}$, et al. "Non-classical" HER2 FISH results in breast cancer: a multi-institutional study. Mod Pathol. 2017;30:227-35.

20. Shah MV, Wiktor AE, Meyer RG, Tenner KS, Ballman $\mathrm{KV}$, Green SJ, et al. Change in pattern of HER2 fluorescent in situ hybridization (FISH) results in breast cancers submitted for FISH testing: experience of a reference laboratory using US Food and Drug Administration criteria and American Society of Clinical Oncology and Coll. J Clin Oncol. 2016;34:3502-10.

21. Donaldson AR, Shetty S, Wang Z, Rivera CL, Portier BP, Budd GT, et al. Impact of an alternative chromosome 17 probe and the 2013 American Society of Clinical Oncology and College of American Pathologists guidelines on fluorescence in situ hybridization for the determination of HER2 gene amplification in breast cancer. Cancer. 2017;123:2230-9.

22. Press MF, Seoane JA, Curtis C, Quinaux E, Guzman R, Sauter G, et al. Assessment of ERBB2 / HER2 Status in HER2 -Equivocal Breast Cancers by FISH and 2013/2014 ASCO-CAP Guidelines. JAMA Oncol. 2019;5:366-75.

23. Liu Z-H, Wang K, Lin D-Y, Xu J, Chen J, Long XY, et al. Impact of the updated 2018 ASCO/CAP guidelines on HER2 FISH testing in invasive breast cancer: a retrospective study of HER2 fish results of 2233 cases. Breast Cancer Res Treat. 2019;175: $51-7$.

24. Xu B, Shen J, Guo W, Zhao W, Zhuang Y, Wang L. Impact of the 2018 ASCO/CAP HER2 guidelines update for HER2 testing by FISH in breast cancer. Pathol-Res Pract. 2019;215:251-5.

25. Murray C, D’Arcy C, Gullo G, Flanagan L, Quinn CM, Quinn $\mathrm{CM}$. Human epidermal growth factor receptor 2 testing by fluorescent in situ hybridization: positive or negative? ASCO/College of American Pathologists Guidelines 2007, 2013, and 2018. J Clin Oncol. 2018;36:3522-3. 